\title{
LOGO CREATION ALGORITHM
}

This article is dedicated to corporate design and its impact on online users. It contains definitions of basic concepts and terms of corporate identity. Particular attention is then paid to corporate design and its features - name of the company, slogan, logo, corporate colors, typography, mascot and signature tune. In the main part of article we present the algorithm which should help small and starting online companies to create their own logo without help of professional designers.

Keywords: Logo, corporate identity, logo creation algorithm.

\section{Introduction}

Every day, we come across hundreds of different signs of companies' activities. It is not only products that meet customer needs. Companies have to differentiate and identify themselves. Even bakeries use specific signs on the breads to ensure customers that they will get the quality and the taste they have expected. Sum of all the phenomena that distinguish the company from its competitors is called corporate identity. According to the most widely used definition, we can identity three parts of corporate identity [1]:

- corporate behavior,

- corporate communication,

- corporate design.

Some authors present the fourth part of corporate identity - corporate product [2]. Although product is one of the core stones of the marketing, we also consider it as a part of corporate identity. There are dozens of examples that prove that corporate product should be considered as a part of corporate identity. Therefore we decided to use the definition of corporate identity that consists of four parts and besides corporate culture, corporate communication, and corporate design it contains corporate product as well.

\section{Corporate behavior}

Corporate behavior is better known as organizational culture. Employees of the company form it and it consists of the values and norms that they share. It also contains standards of the behavior. How the doorman greets you, how the secretary answers your phone, all of this is considered as a part of corporate culture
[3]. Basically we can define two types of corporate culture strong and weak one. Company with a strong culture has better position on the market because employees believe in the values of the company and are willing to follow its mission and vision. Company with weak corporate culture has problems to keep the alignment with organizational values. In such cases employees are not dedicated to the company and control must be secured with red tape.

\section{Corporate communication}

Corporate communication is the set of tools and methods used by company. It enables smooth flow of information. Communication can be internal or external. Both types of communication are crucial for successful operation of the company. Without effective communication there is lack of understanding between different stakeholders. Employees do not know and do not understand where the company is heading, managers do not know where the plugs in production are; customers do not know what the company is offering. Therefore corporate communication cannot be neglected.

Internal communication refers to communication within the company. There are three possible directions of information flow: $\mathrm{Up}$, down or horizontal. First type directs information from lower levels of the organizational structure upward. Second type directs information flow opposite way, from the top to the bottom of the organizational structure. Last type, horizontal communication enables fellow workers on the same organizational level to share their knowledge and experiences.

External communication refers to communication outside the company. It includes communication with investors, government, institutions (such as insurance company), suppliers,

\footnotetext{
* ${ }^{1}$ Radovan Madlenak, ${ }^{1}$ Lucia Madlenakova, ${ }^{2}$ Jana Strbova, ${ }^{1}$ Jozef Stefunko

${ }^{1}$ Department of Communications, University of Zilina, Slovakia

E-mail: radovan.madlenak@fpedas.uniza.sk

${ }^{2}$ EET Online Marketing Coordinator, Lenovo, Czech Republic
} 
intermediaries, public and customers. Therefore, for example, marketing communication is considered as a part of external communication. The same is with public relation [4].

\section{Corporate product}

Product is anything that can be offered to the market to satisfy customer's needs and wants. It can be tangible or intangible. Therefore service such as insurance is considered to be product as well. Corporate product is what customers are looking for. Therefore it is the best way how to prove that certain company is the best choice for them. Customers make their decision according to their individual expectations. If the product brings them higher value than they have expected, they are happy, and vice versa. We can define four levels of product:

- generic product - is the core product. It is the most basic form of the product. On this level products from different producers are generally the same. Customers expect more than just generic product;

- expected product - consists of the core product and other features, which together satisfy customers expectations. In other words, expected product is what customers buy to fulfill their needs and wants;

- augmented product - consists of the expected product and extra features, which overcome customer expectations. This is the level that enables companies to differentiate the product by offering something extra. Today, expected product is enough level for differentiation. Customers actually look for additional benefits;

- potential product - represents all possible extensions which can be offered to customers. Augmented product consists of real and working attributes while potential product is the promise to the future [5].

Customers perceive product levels differently. What is an expected product for one person is an augmented product for another. The bigger customer expectations, the more difficult for the company to meet them.

\section{Corporate design}

Corporate design consists of all elements that create unified presentation of the company. It includes graphical, textual but also other elements [6]. We can define these:

- name of the company is a very important element of corporate design. It is also the name under which the company is registered in a trade or professional register.

- slogan - The Americans use the term tagline or tag, while in UK it is more often to say endlines or straplines. All the time it refers to few words or a sentence which complement company name. It can also be connected with a certain product or commercial campaign. When the slogan is good, customers automatically connect it with the company.

- color is the visible spectrum of electromagnetic radiation with wavelengths around 400-700 $\mathrm{nm}$. Just as this definition, the whole science around the use of colors as a part of corporate design is very extensive. Color is an excellent way how to differentiate company.

- typography - Word typography has its origin in Greek. It

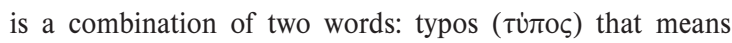
form and graphe ( $\gamma \rho \alpha \phi \dot{\eta})$ that means writing. Therefore typography deals with the form of writing.

- mascot is used to create positive attitude towards the company.

- signature tune is not that widely used because not all companies need it. But when it is done in such a great way as Nokia did with its ringtone, it is heard about 20000 times per second worldwide. And this makes significant contribution to differentiation of the company.

- $\log 0$ is very often mistakenly confused with the trademark. However, we have to realize they are not synonyms. Logo is the graphic representation of the company name. It does not necessarily have to contain the name but it should refer to it. When it contains only a picture, it is called pictogram. When it contains only graphic transcription of the name, it is called logotype. Pictogram together with logotype creates logo.

\section{Aim and methodology}

Dozens of different corporate designs bombard us every day. We cannot avoid it. These designs carry hidden messages whether we see it or not. Our subconsciousness feels more than is visible with the naked eye. Colors, shapes and even fonts affect our perception of the $\log 0$ as a part of corporate design.

We have many examples of good and also bad logos, of different colors, shapes and fonts. How they affect us and in what way they should be used. Of course every logo has to be tailor made according to the company. However, sometimes it is not done very well. Companies like Amazon or Martinus have the capital to hire professional designers or even announce contest for the best logo. But there are many small companies which have just started to do the business and cannot afford it. They have to create the logo on their own. Unfortunately they usually create the logo according to their preferences. If they like black color they simply use it no matter that they are running a toyshop. Wrong font can also cause misunderstanding of logo. And using wrong shape can cause real disaster.

However the main goal of this article is to help small and starting companies to clarify basic principles how to create their logo. Using our created methodology can help them to orient themselves in the process of logo creation. We hope that this logo creation algorithm will be helpful for commercial and also other purposes. 


\section{Results - $\log 0$ creation algorithm}

To make it even easier for companies, we created algorithm that includes different situations that may arise, how they can be resolved and what procedures should be followed.

We divided the algorithm into six different parts that are also differentiated by color. The most important is the beginning and the end of the algorithm, which are represented by red ellipses. Prerequisite for the algorithm application is the decision to create logo. When this decision is made, we can proceed to the process of $\log 0$ creation.

First, we have to have the company name. This part of algorithm is differentiated by grey color (Fig. 1). If the company has its name, we can proceed to the second part. If not, the company name has to be created. This is especially true in the case of starting companies that are not running their businesses yet.

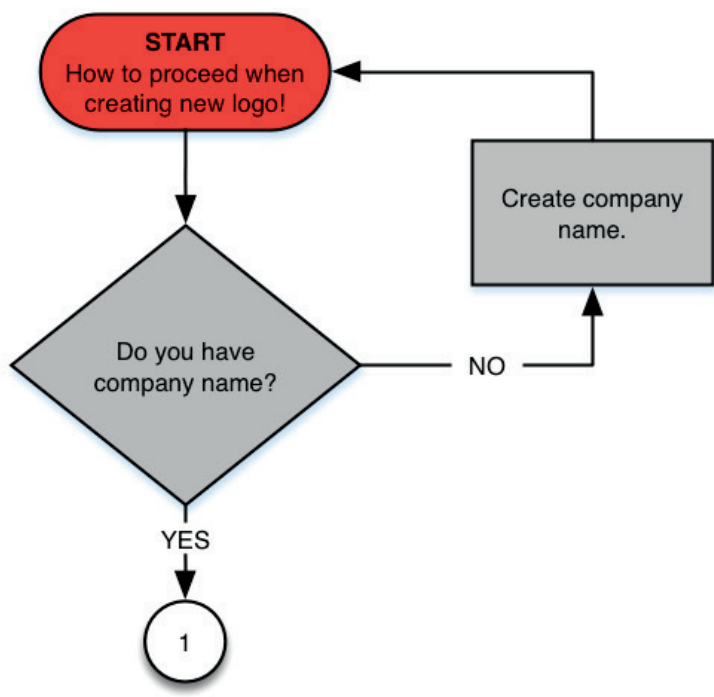

Fig. 1 Logo creation algorithm - first part (Source: Authors)

Second part of algorithm is green (Fig. 2). It describes the process of picture or symbol implementation into the logo. At the beginning we have to identify the core business. If we can define such an area we have to find a picture that represents it. If there is no picture that represents the core business, we have to find the picture that represents company name. The same is also done in the case we cannot define core business. If there is no possibility to find a picture that is connected with the core business or company name, our last chance is to look for the motive in mission or vision of the company or anything else that is connected with the company. If none of this is possible, we should use the solution without picture - logotype. As we have already mentioned, logotype is type of logo where no picture or symbol is used. It is the logo purely designed from the company name. But there are still great and significant logotypes that are engraved in minds of customers. Just think of Coca Cola or Google [7]. No need to implement picture. And still very well spoken. If we have identified some picture or symbol connected with the core business, company name or its mission or vision, we have to decide whether it is possible to implement it into the name. Sometimes there is opportunity to replace one character from the company name by the picture. This has to be considered very carefully and the picture has to fully replace the missing character. The name has to be easily readable after this change.

If there is no chance to replace the character by the picture or we decide not to use this opportunity, we have to find the best place for the picture next to the company name. Of course, in this part we are not limited and we can create more samples of logo. For example, with different picture placement. This part of algorithm is done when samples of logo or logotype are created.

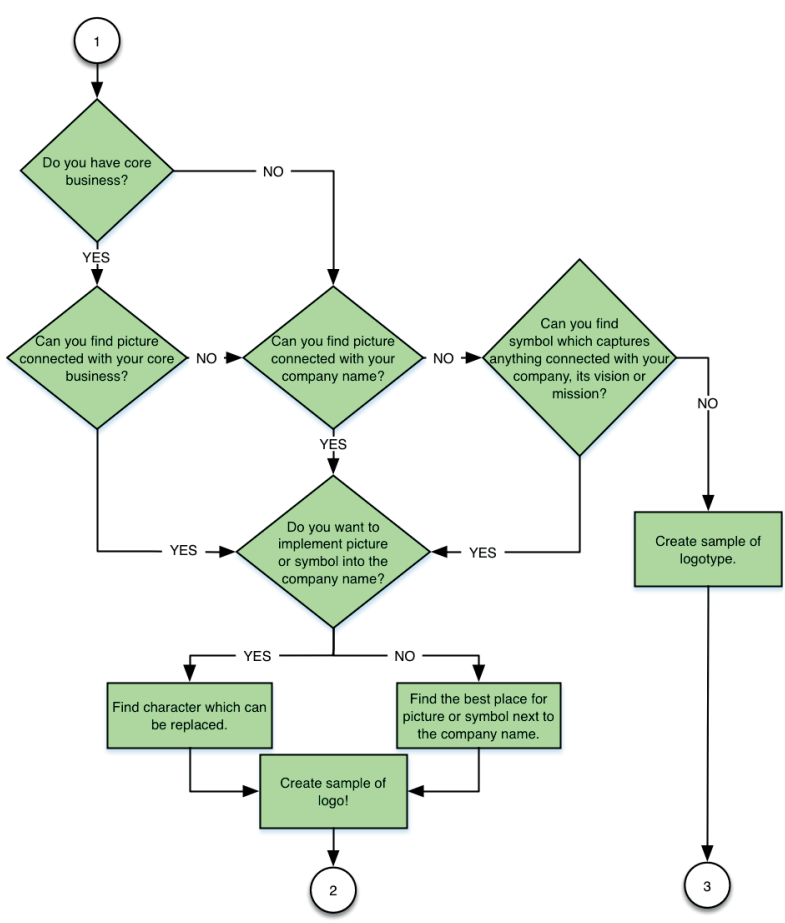

Fig. 2 Logo creation algorithm - second part (Source: Authors)

Another part is differentiated by orange color and is dedicated to fonts (Fig. 3). Font is integral part of $\log 0$ and corporate identity as well. Chosen font should be used not only as a part of the corporate design, but also as a part of overall identity letterhead paper, newsletters and other documents as well. And, of course, we cannot omit websites. At the beginning of this part of algorithm we have to try different fonts and choose few that go well with our logo samples. The number of fonts does not limit us. 
However the more fonts the longer it takes to analyze them. When we have chosen the right fonts we have to analyze them. First, we have to identify to which group they belong. Whether they are from geometric, humanist, old style, transitional, modern or slab serifs group. If we do not know to which group they belong we have to find out. After this is done we have to explore the hidden meaning of the fonts. Try to go deeper, associate font with your company. Each group contains dozens, even hundreds of different fonts and they carry their special message as well.

When we are done with hidden meaning analyzing, we should eliminate fonts that do not meet our requirements. Of course, if we are not satisfied with the results, we can go back at the beginning of this part of algorithm and try to use different fonts.

At the end of this part we should have samples of logo with chosen fonts. Of course, it does not have to be necessary one font. We can proceed with samples that contain different fonts and different pictures as well (or only different fonts in the case of logotype).

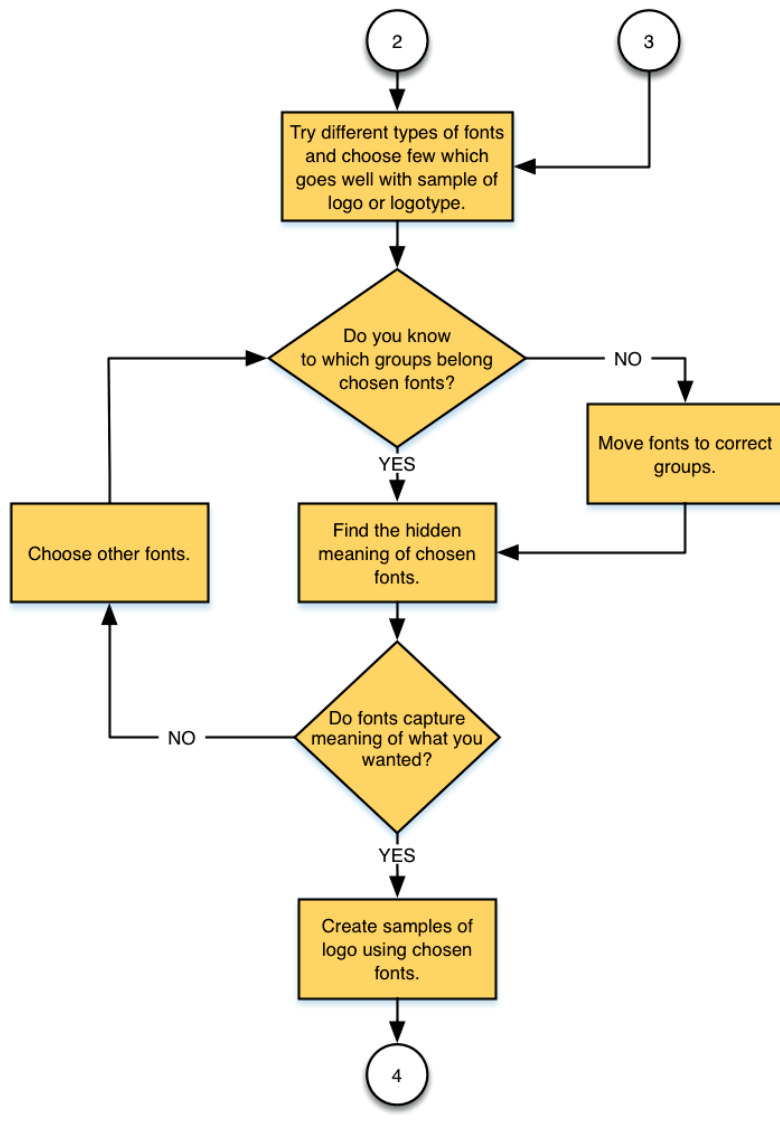

Fig. 3 Logo creation algorithm - third part

(Source: Authors)

Blue part of the algorithm is dedicated to colors (Fig. 4). Colors have significant impact on our subconsciousness. How strong? Well, think about the angry bull when it sees a red scarf.
Of course, this is an example from animal life, however red color has impact on human blood pressure as well. And other colors have impacts too, just in a different way [8]. Therefore, we have to think very carefully with which colors we connect our company. Process of colors selection is similar to font selection. At the beginning we have to try different color combinations on different logo samples. In every step of the algorithm we can eliminate the sample that just does not seem to be the right one. When we are done with choosing different colors, we have to reveal their hidden meaning.

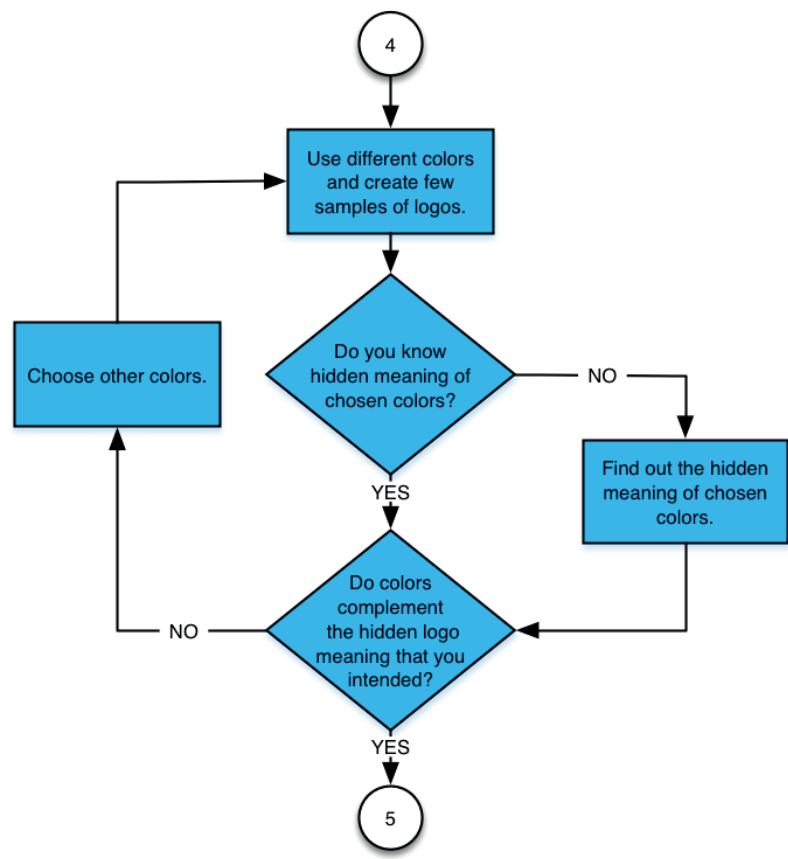

Fig. 4 Logo creation algorithm - fourth part

(Source: Author)

Once again, colors that do not complement the overall meaning of logo that we have intended should be eliminated. Only colors that go well with our samples of logo should proceed to the next part of algorithm.

Next part of algorithm is sample testing and it is differentiated by violet color (Fig. 5).

Our logo samples, which consist of company name, picture or symbol and chosen colors (in the case of logotype without pictures) have to be tested. So far we have done all the work. Now it is time to involve other people in this process as well. We should choose the samples that fulfill our requirement best. These samples should be introduced to our potential customers. In the business plan we have to identify who our potential customers should be, therefore, we know whom to ask. We can find them via social media or other online tools. If we know them directly we can even ask them personally. There are also different websites that are supported by designers. We can ask them about their professional opinion as well. There are no limitations in this part 


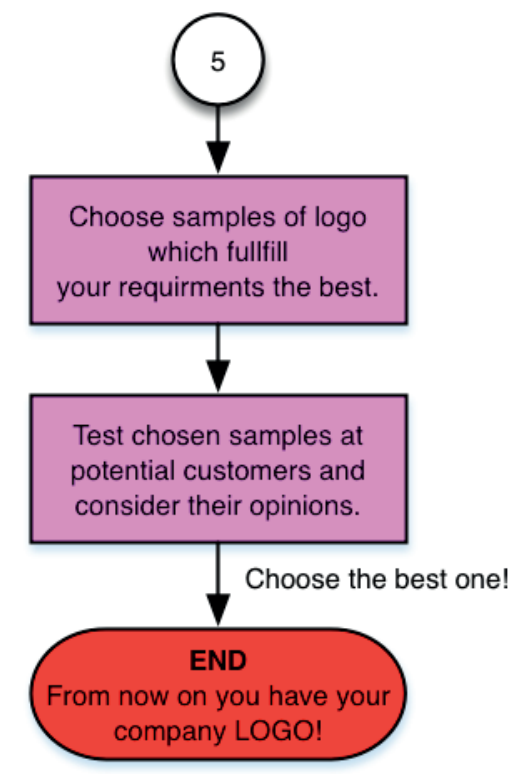

Fig. 5 Logo creation algorithm - fifth part (Source: Authors) of algorithm, we can try as many different ways as possible and gather all the different opinions and attitudes. By analyzing them we can decide which logo represents our company best. The one that is interpreted by customers the way we intended.

When all of this is done, we are at the end of the algorithm - red ellipse that signalizes that we have created our new logo!

\section{Conclusions}

Design and creation of the logo is a very important process. In this article we tried to compose the universal algorithm that will help small and medium companies with this problem. We hope that this proposed logo creation algorithm would be helpful for commercial and also other purposes.

\section{Acknowledgements}

KEGA-053ZU-4/2013 - Improving and Linking the Key Subjects of the Study Program Ecommerce and Management.

VEGA $1 / 0421 / 12$ - Modeling diffusion of knowledge in corporate value chains.

\section{References}

[1] VAN RIEL, C. B. M., BALMER, J. M. T.: Corporate Identity: The Concept, its Measurement and Management. European J. of Marketing, [online]. 1997, vol. 31 No. 5/6. [Quoted 2012-02-02]. Available at: http://repub.eur.nl/res/pub/12137/Corporate Identity_1997.pdf, ISSN 0309-0566.

[2] KRIEK, Z., CRHA, I.: How to Write Advertising Copy. 3 updated edition. Praha: Grada Publishing, 2008, pp. 116-117, ISBN 97880-247-2452-2.

[3] HILL, Ch. W. L., JONES, G. R.: Strategic Management: An Integrated Approach. $8^{\text {th }}$ edition. Boston: Houghton Mifflin Company, p. 416, 2008, ISBN 978-0-547-00497-6.

[4] Business Communication and its Types, [online]. [Quoted 2012-02-09]. Available at: <http://www.rizwanashraf.com/2008/02/04/ business-communication-and-its-types/>.

[5] BACON, T. R., PUGH, T. G.: Winning Behavior: What the Smartest, Most Successful Companies Do Differently. New York: AMACOM, American Management Association, p. 16-18, 2003, ISBN 0-8144-7163-3.

[6] WANG, Q.B.: Statistical Analysis on the Enterprise Logo Color Designs of Global 500; in: 10th IEEE International Conference on Computer-Aided Industrial Design and Conceptual Design, Wenzhou, China, p. 277-280, 2009, ISBN: 978-1-4244-5267-5

[7] LIU, X., ZHANG, L.: The Study of Color in the Design of Brand Trademark; in: International Conference on Information Technology and Scientific Management, Tianjin Polytechn Univ, Tianjin, China, 2010, p. 276-278, ISBN: 978-1-935068-40-2

[8] ADIR, G., ADIR, V., PASCUC, N.E.: Logo design and the corporate identity; in: World Conference on Design, Arts and Education, Antalya, Turkey, p. 650-654, 2012, ISSN: 1877-0428. 